\title{
A preliminary exploration of attitudes about COVID-19 among a group of older people in Iwate Prefecture, Japan
}

\author{
Lauren $\mathrm{He}^{1}$. John W. Traphagan ${ }^{2}$
}

Accepted: 15 January 2021 / Published online: 9 February 2021

(C) The Author(s), under exclusive licence to Springer Science+Business Media, LLC part of Springer Nature 2021

\begin{abstract}
In this article, our goal is to provide researchers with a non-western perspective concerning the COVID-19 pandemic through a qualitative study of older adults living in Iwate, a prefecture in Japan that has only recently (27 July 2020) reported its first confirmed COVID-19 cases and which has experienced comparatively few cases since. Because they live in an environment with few COVID-19 cases, the individuals in this study provide an interesting perspective of the pandemic as it is unfolding in rural Japan and our focus here is on documenting the circumstances and ideas of elder residents of Iwate Prefecture to understand individual experiences of this population. Our intention here is to provide data and tentative analysis that may be useful in developing more complex cross-cultural studies related to the lived experience of COVID-19 among older people.
\end{abstract}

Keywords COVID-19 $\cdot$ Older japanese $\cdot$ Pandemic $\cdot$ Rural elders

\section{Introduction}

From its first appearance in Wuhan, China as an unexplained pneumonia in December of 2019 (Zhu et al. 2020), COVID-19 has now infected more than 91,000,000 people and is attributed to nearly 2 million deaths worldwide (World Health Organization 2021). It has become evident that the older population is more susceptible to the disease, with patients observed to develop more severe symptoms and a higher mortality rate (Liu et al. 2020a, b). Therefore, the impacts of COVID-19 on older people has become a topic of urgency, and a growing body of research is emerging related to this population.

John W. Traphagan

jtrap@utexas.edu

1 Westlake High School, Austin, TX, USA

2 Department of Religious Studies, University of Texas At Austin, A3700, 2505 University Ave, Austin, TX 78712, USA 
Several studies have focused on biomedical analysis of COVID-19 for older patients in order to investigate clinical characteristics of those with COVID-19 (e.g. Liu et al. 2020a, b; Wang et al. 2020a, b; Zuo et al. 2020; Niu et al. 2020; Guo et al. 2020). Studies have also explored the potential consequences of social distancing on elder well-being, emphasizing the indirect detrimental impacts of COVID-19 and the need to address them (e.g. Armitage and Nellums 2020; Wand et al. 2020; Patel and Clark-Ginsberg 2020). From a social science perspective, behavior and opinion changes through the timeline of COVID-19 have been reported through analyzing survey data to observe differences between age groups (e.g. Di Renzo et al. 2020; Barber and Kim 2021). A gap in this literature can be found in the understanding of COVID-19 from the perspectives of elders themselves. In qualitative studies done on individuals' experience with COVID-19 in general, the content is more focused on the psychological experiences of healthcare providers and caregivers, rather than patients or elders who themselves are at higher risk of significant negative impacts from COVID-19 (e.g. Liu et al. 2020a, b; Munawar and Choudhry 2021; Sun et al. 2007, 2020).

In this article, our goal is to provide researchers with one set of perspectives concerning the COVID-19 pandemic through a qualitative study of older adults living in Iwate, a prefecture in Japan that has only recently (27 July 2020) reported its first confirmed COVID-19 case and as of this writing there have been only 23 cases in the prefecture with zero deaths (Sasaki and Nishizuka 2020). Because they live in an environment with few COVID-19 cases, the individuals in this study provide an interesting perspective of the pandemic as it is unfolding in Japan and in relation to other countries and our focus here is on the circumstances and ideas of elder residents of Iwate Prefecture to understand individual experiences of this population. To our knowledge, this is the first qualitative study done on the COVID-19 situation from the perspective of individuals living in Iwate.

\section{The Setting}

Japan was one of the earliest countries to be exposed to COVID-19, having identified its first case on 15 January 2020 (World Health Organization 2020). It is worth noting while Japan's proportion of older adults $(65+)$ is among the highest in the world, compared to countries with similar demographics, it has managed to keep its COVID-19 infection and death numbers relatively low (Khafaie and Rahim 2020). As noted above, Iwate Prefecture, located in the northeastern part of Honshū, Japan's main island, has been unusual for its lack of confirmed cases of COVID-19 until July 27. Awareness of this situation, as a result of ongoing contacts with local residents and one of the authors, led to questions related to how people living in the area might conceptualize their unusual situation as it relates to the overall global pandemic, as well as other parts of Japan that have experienced higher rates of infection. Early conversations with interlocutors in Iwate revealed some interesting concerns, including perceptions of a strong desire among residents to be extremely careful about taking precautions against getting and spreading the disease out of a desire to avoid being the "first case" in the 
prefecture, which several individuals indicated would be embarrassing because it would suggest that the infected individual(s) had not taken sufficient precautions to avoid the disease and, thus, put others at risk. This is quite consistent with attitudes about responsibility for self-care and the onset of disease among older people living in Iwate (cf. Traphagan 2000).

Ōshū City, where the participants in this study reside, has just under 115,000 residents, representing a decrease of approximately 10,000 people since 2010 (Traphagan 2020). As of 2020, 35 percent of the population is over the age of 65 and this proportion is expected to continue to increase significantly over the next several years reaching a high of 44 percent by 2045 (NIPSSR 2018). The region consists of a mix of agricultural and industrial sectors, although most local residents characterize the region as somewhat traditional from a values-perspective in contrast to perceived urban and cosmopolitan areas like Sendai, a city of about 1 million in the neighboring Miyagi Prefecture just south of Iwate, and Tokyo, which is about $600 \mathrm{~km}$ to the south. Nonetheless, many residents have travelled extensively and many participants in this study have experiences with overseas travel. Thus, the people who participated in this study should be understood as being integrated into global cosmopolitan flows of goods and ideas (Traphagan 2020).

It will be instructive to briefly discuss the structure of the Japanese healthcare system, because it differs significantly from that in countries like the US, which have had much higher rates of COVID-19 infections and deaths than Japan. Japan has a mandatory national health insurance system in which all citizens are covered, as well as many foreign nationals living in the country (Matsuyama 2014). Cost sharing varies in relation to age, with adults typically paying $30 \%$ of costs for medical expenses until the age of 70 , after which the copayment drops to $10 \%$ and there is an out-of-pocket threshold that protects individuals from catastrophic costs related to healthcare (Japan Health Policy NOW n.d.). This system is augmented by a mandatory national long-term care insurance program that provides services for older people, such as home helper and nursing home services and residential group homes for those suffering from dementia (Traphagan and Nagasawa 2008). The combination of government run programs with the fact that fees are regulated by the government means that Japan has a comparatively low-cost healthcare system with one of the most equitable access structures in the world (Ikegami 1991). This is important to keep in mind as we work through the data presented here, because the overall context of the Japanese healthcare system certainly influences the ways in which participants in this study responded to our questions. Unlike the US, the people with whom we spoke here assume that everyone will have full access to all necessary medical care should they contract COVID-19. This is a reasonable assumption given the fact that Japan has a universal health insurance system and also uses a public health model that has local public health centers, in addition to private clinics, which participate in testing, patient triaging, contact tracing and allocating COVID-19 patients to designated isolation hospitals (https://www.hsph.harvard.edu/news/hsph-in-thenews/lessons-from-japans-covid-19-response/). In short, Japanese are accustomed to expecting treatment for all healthcare needs that fall within the national health insurance scheme. 


\section{Methods}

This qualitative study employed both a single focus group session and a brief questionnaire. ${ }^{1}$ Thematic analysis was used in order to explore common themes of the participants' subjective thoughts, perceptions, and experiences of COVID-19. Due to current travel restrictions, data were collected via a combination of online survey (using Google Forms) and an informal focus group meeting arranged with an English language class located in Ōshū City, Iwate Prefecture, and that was conducted via Zoom and lasted approximately $1.5 \mathrm{~h}$. The participants in this study were volunteers who take an English class conducted on a weekly basis at a local international center that is led by a local resident who is a native speaker of Japanese and fluent in English. One of the researchers (Traphagan) is fluent in Japanese. This combination allowed for interpreting to occur in cases where class members struggled to understand a question or to convey their thoughts adequately in English. Sampling was nonrandom and can be understood as a convenience sample in that we focused on the opportunity provided by our connections to the English class. This is reasonable, as our intention is not to generalize to a larger population, but to gain insight into the individual experiences of people living in Iwate and, in particular, to develop an initial sense of local explanations for why Iwate managed to avoid any reported COVID-19 cases until several months into the pandemic. Part of our interest was in exploring how older individuals living in Iwate may employ cultural explanations for their unusual situation.

On 21 July 2020, the authors conducted an informal focus group meeting via Zoom during which participants in the class answered a series of open-ended questions in English. The meeting was arranged as a means of obtaining subjective data from participants about COVID-19, as well as an opportunity for participants to practice their English skills with native speakers (the researchers). The class consisted of 11 individuals with an average age of 61.73 (with a high of 68 and low of 56). One of the authors (Traphagan) has conducted ethnographic research in the city and some of its neighboring towns since the 1990s; as a result, participants were identified through an established contact from previous, in-person field trips, the class teacher being one of Traphagan's regular interlocutors (Traphagan 2000, 2004a, b, 2020). Most of the participants used English, but those who chose to speak in Japanese had their responses translated by the class teacher. A survey was sent out to the same group on 4 August 2020 that contained more specific questions regarding elderly lifestyle changes during COVID-19. The survey included both multiple choice and open-ended questions in order to give participants the opportunity to provide context and elaborate on their ideas from the interview and was returned by 10 August 2020.

\footnotetext{
1 Note that this study did not undergo the normal IRB process, because the lead author's institution (a high school) does not have an IRB. All normal protocols for human subjects research were followed and informed consent was obtained from participants. All participants were made aware that participation was completely voluntary and that they could withdraw at any time.
} 
Because our sample is small and nonrandom, the results should not be taken as representative. However, based on several years of ethnographic research in the area by Traphagan, we believe that it is reasonable to conclude that the perspectives offered by the participants in this study are not atypical of those among people in similar age groups living in Ōshū and its surrounding municipalities. In the surveys, participants were directed to two paths, depending on whether they self-identified as being over or under the age of 60 . Those over $60(n=7)$ were asked questions that referred to their personal experiences related to COVID-19, while those under $60(n=4)$ were asked to respond to questions about someone they know over the age of 60 . Therefore, the number of referents in the study is also 4, with an average age of 85.25 (with a high of 87 and low of 84). Participants in the study largely live in free-standing homes, while older individuals referred to by those under 60 live in a combination of free-standing homes $(50 \%)$, apartments $(25 \%)$ and nursing care facilities $(25 \%)$. There is an array of family situations for both participants and referents that include living alone, living with adult children, living with a spouse only, and living with a parent only. The traditional pattern of the three-generation family associated with rural Japan was encountered in only one response, which is reflective of long-standing changes in living arrangements of older people in Japan over the past fifty years in which a significant proportion of the older population has come to live in either single or couple elder households (Traphagan and Knight 2003).

In general, responses showed only limited change in behavioral patterns among both participants and referents in the study (see Table 1). For example, when asked about the frequency of leaving home by the individual referred to among those under $60,75 \%$ indicated that there had been no change between pre-COVID behavior and behavior during the pandemic. Prior to the pandemic, respondents indicated that $50 \%$ of referents only left the house about once-a-month, while $25 \%$ left every day. This is likely associated with the fact that some referents were living in nursing facilities and also with the fact that it is not uncommon for older people to rarely leave the house in rural parts of Japan. By contrast, those over the age of 60 in the study who responded to the survey about themselves indicated a much higher frequency of meeting with family and friends before the pandemic, all responding that they met daily or several times a week. Furthermore, the majority $(85.7 \%)$ of participants in this group indicated that the frequency of meeting with family and friends had not changed since the start of the pandemic. Similar patterns were observed in questions related to frequency of leaving the home, though a few individuals were more inclined to stay home during the pandemic. Overall, the survey data we collected indicated relatively limited changes in behavior as a result of the pandemic, largely being restricted to maintaining social distance, wearing masks, and frequently cleaning hands and surfaces. This will become more evident as we move through the qualitative focus group data discussed in the following sections. 







\section{Focus Group Outline and Analysis}

The focus group questions were broad and open-ended to ensure participants could share their thoughts and perceptions of COVID-19 easily. In constructing the questions, emphasis was placed on learning about the lives and perspectives of older people and the unique situation in Iwate (i.e. no confirmed COVID-19 cases at the time of the focus group meeting). We were also interested in exploring the ways in which locals interpret the fact that Iwate went for several months without any reported cases and that Japan as a whole appears to have fared relatively well in controlling the spread of the virus as compared to contexts like the US and the EU. We were also interested in the extent to which our participants may have attributed this to cultural factors connected to the region or to Japan. The primary questions asked were the following: "what are some reasons you think Japan's confirmed cases of COVID-19 and deaths are low compared to other countries"; "what do you think is unique about Iwate that has contributed to no confirmed cases of COVID-19 yet", "what are some ways the community came together to help protect those most vulnerable, specifically the older population"; and "what are aspects of daily life for older adults that have changed the most?" It is important to keep in mind that these questions were presented largely in English (with occasional interpreting by the teacher of the class) and were designed to be as easily understood by non-Native English speakers as possible. Also, as part of the English experience for the members of the class, they were encouraged to respond only in English, although several switched to Japanese when they found it difficult to convey their ideas clearly in English.

The focus group session was recorded using Zoom with the consent of the participants and transcribed afterwards. Data were explored through thematic analysis, in which, after familiarizing with the data through reading multiple times, significant phrases were identified, compared, and sorted into key themes. As noted above, participants in the focus groups were also asked to complete a survey, in English, that was presented to the group approximately 1.5 weeks after the meeting via Zoom. The purpose of the survey was to allow participants to elaborate on prior comments (given that English is not their native language) and also further pursue questions related to how the pandemic has influenced the lives of older people living in Ōshū. Responses to the survey's open-ended questions were compared to the existing interview data and themes. Descriptive statistics were used to summarize quantitative data and provide characteristics of the participants previously detailed.

\footnotetext{
${ }^{2}$ The authors acknowledge that this question, in particular, might be viewed as leading. However, given the unusual nature of the lack of COVID-19 in Iwate-at the time it was the only prefecture in Japanwe decided that it was important for participants to discuss why they believe the situation in Iwate was unique in comparison to other parts of the country. It is a topic that was being widely discussed by residents at the time.
} 


\section{Results}

Several interesting themes that emerged from analysis of the focus group and openended survey questions. Responses generally fell into the following thematic categories, most of which convey ideas reflecting cultural patterns either associated with Japan or with the more local context of Iwate:

- Caution and preparedness

- Thoroughness and attention to detail

- Recognition of the necessity of sacrifice

- Relationships with others in society

- Unique disposition of people

- Attention to cleanliness

- Collective experiences

We will work through these themes in the following discussion, drawing on comments from the focus group session. Most of the comments emphasized reflections on the ways in which participants see their own culture in relation to Japan in general and more specifically in relation to Iwate Prefecture. We will work through these by assigning broad category names with subthemes that were evident from the perspectives of the researchers in each category.

\section{CATEGORY 1: PRAGMATIC THEMES}

\section{Caution and Preparedness}

As has been noted by Traphagan and others, Japan has a well-developed system of home help services designed to support older people living independently and this is considered important among our participants in explaining the general lack of spread of the virus in the region (Traphagan 2004b). Home help services have been supporting the elderly living independently even before COVID-19 and it is reasonable to consider the possibility that this existing service could contribute to the care system already being relatively well-prepared to help older people who lack the ability to regularly leave their homes. As one individual indicated, "We have many elderly services for shopping, for food, meal services as well. So the older...so they don't have to go out." ${ }^{3}$ Closely tied to this was an emphasis on shared actions that reflect precaution that are often evident through care and help services, especially when working with older people. For example, strict procedures and safety measures are followed in home help services such as provision of in-home baths, where there is considerable attention given to sanitization as well as provision of regular

\footnotetext{
${ }^{3}$ Note that we have quoted English exactly and because the participants in our study are not native speakers, there may be grammatical errors. We have chosen not to correct these errors in order to avoid changing any meanings that may be represented in comments.
} 
health checks through taking blood pressure and temperature readings before a bath is given or simply when visiting an older person's home (Traphagan 2004b). This is reflected in one participant's response: "We have home help services, and they have to check their temperature everyday in the morning, and they have to wear masks and also special aprons and everything, scrubs, so they visit elderly people's homes and support their lives." In general, responses to this theme indicated a sense that the presence and easy availability of services, which are part of the national health insurance and long-term care insurance programs, allow close monitoring and reinforcing of practices designed to limit the spread of the disease. In addition, most of the practices associated with reducing the spread of COVID-19 long have been practiced among care workers in the field of home help services in Japan.

\section{Thoroughness and Attention to Detail}

While describing elder care, participants also noted thoroughness in the procedures taken. One participant explained her experience with nursing care services: "They check temperature every hour... and are very, very careful...They have to close the facility even if one old people or staff tests positive of COVID-19." Other participants in the study mentioned this attention to detail and thoroughness as being important in taking measures against the spread of COVID-19. One participant mentioned the wide use of cleaning supplies and disinfectants and the ready availability of these supplies in most stores and other public spaces-the general sense was that places and individuals are prepared with necessary supplies, and because of this, they can take precautionary measures against COVID-19 regardless of where they go in their daily travels. As one participant explained, "I think alcohol... [alcohol, alcohol]... is prepared in many places, department stores and supermarkets, for example the library. Many people bring mini-sized alcohol, or paper towels, wet towels, many people have...everywhere."

\section{Recognition of the Necessity of Sacrifice}

Precautionary measures generally taken by older individuals include using products such as hand sanitizers and limiting travel to avoid close contact with others. Although practical efforts-such as making disinfectants widely available and the presence of well-developed help and care services, may ease the difficulties older individuals encounter when practicing precaution-there are perceptions of tradeoffs being made in order to reduce spread of the virus. Some participants noted the impact of social distancing practices on social relationships while acknowledging the necessity of decreasing social relationships: "I changed my life. I can't see my children and grandsons... they live in Tokyo. Tokyo has many COVID-19 cases... I told them don't come back home until COVID-19 is done."

Another participant stated, "my mother-in-law lives in a nearby prefecture. She lives in a welfare facility for the elderly. Tomorrow, I was going to visit my motherin-law, but yesterday, facility staff called us: 'please no visit.' I look forward to meeting my mother-in-law in four months.... yesterday somebody told me 'no visit'. 
To protect, I think, her, but it's good for others. That's the way it is." This woman's comments reflect broader Japanese emphasis on being aware of the needs of others, as well as a certain sense of resignation to the fact that the situation is what it is, making it necessary to accept the inconvenience of not being able to visit her mother-in-law. It is a sentiment that reflects a widely used Japanese phrase, shikata ga nai, which basically means "it can't be helped" and refers to situations in which events or conditions are beyond one's immediate control and, therefore, simply must be accepted (Matthews 1996:723).

Finally, participants recognized the negative impacts on mental and physical health, which was particularly important in relation to older people, because the current situation means that older people cannot go to day service centers or public/ private facilities that provide opportunities to exercise safely and socialize with agepeers (Tsukada and Saito 2006). The limited availability or hesitation of older people to visit day service centers for older people during the pandemic has corollary problems indicated in the concerns of some participants about the hesitancy of older family members or neighbors to seek medical attention when needed: "In my area, who living in older people [where there are many older people living], they have been staying home. They don't have so much exercises, so they have pains on the legs, less communication...Most elderly people don't want to go out, even go to see a doctor, so manage of posture is getting worse. It is problem." In other words, there was concern conveyed among participants about older people experiencing nonCOVID-19 related health problems due to the fact that they cannot or are unwilling to participate in contexts such as day service centers for elders that provide opportunities to exercise and also to interact socially with age-peers.

\section{CATEGORY 2: CULTURAL THEMES}

\section{Relationships with Others in Society}

Participants generally agreed that people in Japan strive to maintain social order. One key factor indicated in ensuring that this happens is value placed on the individual responsibility to not bother others. In the context of COVID-19, the main action to take in order to not be a burden on others is to proactively try to avoid getting infected. The reasoning is that if one does not have COVID-19, then others do not need to worry about being around an infected person. This theme taps into significant value placed in Japanese culture on burden avoidance, which often manifests itself in the form of taking actions aimed at maintaining one's own health in order to avoid becoming a burden on family, neighbors, or government services and is a concept particularly strong among older members of society (Lock 1995; Traphagan 2000). As stated by one participant: "I think the Japanese don't want to bother other people in the society by being infected by the COVID-19." Several participants also mentioned other actions individuals take in society — or in this case, do not take that are related to not causing trouble:

"We don't go to drinking parties." 
"We don't want to bother to other people." (from survey).

"[M]ost.people.dont.go.out.at.night" (from survey).

In general, there was a broad agreement among participants in the study, whether through the survey or the focus group, that individuals have a responsibility to avoid infecting others and this is carried out by avoiding contexts and behaviors in which the virus is more likely to spread. In a separate conversation outside of this study, one of the authors was informed that there had been some problems in Iwate with public shaming of individuals who appeared to be ignoring general expectations about behavior related to avoidance of spreading the virus, suggesting that this is not simply a matter of internal motivation but also of concern over the perceptions of others in relation to personal behavior associated with attentiveness to the pandemic.

\section{Unique Disposition of People}

Participants also mentioned other social or cultural traits perceived as being characteristic of Japanese people in general and people living in Iwate more specifically that might play a role in controlling the spread of COVID-19. Examples of these include:

"Honesty and sincerity. I think the Japanese observe morality without being forced by regulations. The Iwate people are especially honest." (from survey).

"We are carefully People. We keep promise." (from survey).

Several participants indicated that traits like these may contribute to citizens accepting the government's guidelines designed to prevent the spread of COVID-19, which is also tied into perceptions that Japanese people are generally inclined to follow rules.

"I don't want to cause trouble as is, so we wear mask...actively."

"The government says avoid the 3 Cs. 1, close spaces with poor ventilation.

2 , crowded places with many people nearby. 3 , close contact settings such as close range conversations. Many Japanese people keep the rules."

\section{Attention to Cleanliness}

Individuals also indicated the value of cleanliness in Japanese culture, a point that long has been noted by some anthropologists working on illness in Japan (OhnukiTierney 1984). As COVID-19 is an illness, and many efforts to prevent its spread are focused on proper hygiene practices and disinfecting measures, cleanliness may well be seen as a measure of one's response to COVID-19. Participants used strong words when describing experiences that fall into this theme, stressing their belief of the importance of cleanliness during COVID-19, and tended to frame these responses in terms of Japanese cultural or social behaviors.

"When I go out, I wear mask and disinfect by alcohol every time." (from survey). 
"Japanese always pay attention to the hygiene."

"We always wear masks, wash hands, TV urging people to wear mask and wash hand always."

One of the more interesting responses to questions related to why Iwate Prefecture has largely been spared the spread of the virus was tied to the geographical space itself and draws upon images of rural Japan as pristine and clean (Traphagan 2020). Participants often brought up clean air when asked about factors they think may contribute to Iwate's lack of COVID-19 cases and connected clean air to cleanliness in general as a variable associated with reducing the spread of COVID-19 in Iwate as compared to more urbanized regions of the country.

"I think Iwate has great nature...traffic is a little" (from survey).

"Cleanair" (from survey).

"Iwate prefecture area's air very clear".

"We have clean air... they go to enjoy playing ground golf".

This last point, about playing ground golf (which is a game similar to golf played in playgrounds by older people), indicates a sense that the comparatively clean air-free from the impurities found in urban spaces-allows older people to participate in outdoor activities that might be more difficult if Iwate were not a clean natural space. In other words, the natural environment of a rural area like Iwate, in the view of several participants, contributes to controlling the spread of the virus and has made it easier for older people and others largely to continue following their normal patterns of behavior.

\section{Collective Experiences}

Participants took into consideration previous experiences and practices that were not related to COVID-19 but still could be playing a role in shaping the COVID-19 situation. For example, in general, East Asia has seen lower numbers of COVID-19 cases than other parts of the world, which is a point observed by one participant. Instead of referencing culture or policy, which are common reasons expressed in the media, the participant recalled a shared past experience of Asian countries: "Why is the number of people who get sick from COVID-19 so small in Asian countries compared to other countries? This phenomenon has been noticed worldwide. When I was watching the TV, the program talked about this phenomenon. One reason may be that many Asian people have received the BCG vaccine in childhood. ${ }^{4}$ And this vaccine has been national policy of some Asian countries for the long time."

Another participant attributed the low rate in Iwate to the lack of visits from outsiders. He noted that Hokkaido and Iwate have the first and second lowest population densities in Japan, respectively. However, Hokkaido has seen a much larger COVID-19 outbreak than Iwate. This individual noted a characteristic of Hokkaido

\footnotetext{
${ }^{4}$ Bacillus Calmette-Guérin (BCG) vaccine is primarily used against tuberculosis in countries where the disease is common or has been common in the past. It is not used in the United States.
} 
that contrasts with Iwate and that could be a factor in explaining the difference in cases: "Hokkaido is lowest [population density] in Japan, but Hokkaido is five most infection. Why do you think? Because Hokkaido is a popular tourist destination, so there are many movement from outside, all over the world."

Furthermore, in Iwate, collective memory of the Great East Japan Earthquake still lingers in people's minds, and was seen by some as differentiating the response in Iwate from other parts of Japan. As mentioned by one participant: "I think we have a strong sense of crises about COVID-19 because we have experienced the Great East Japan Earthquake”.

\section{Discussion}

This study was conducted in order to gain an understanding of older individuals' perceptions of COVID-19 in Iwate Prefecture as a means of generating an initial example of cross-cultural data related to the pandemic. Results from the survey conducted revealed information regarding lifestyle changes during COVID-19, although participants answering about their own lives felt as though they had not made many major lifestyle changes during COVID-19 aside from taking the added precaution of wearing masks, disinfecting surfaces, and decreasing social interactions. They continued to go shopping, exercise, and eat at restaurants, and the main activity they discontinued was traveling. Among the participants answering questions about an older person they know, every participant responded "same as before" when asked about the activities the person continued to do during COVID-19, although it was also noted that access to day services for older people had been limited, and this did cause some lifestyle changes among older people referenced. The general consensus among participants in the study was that the lives of older people have not changed drastically, except for taking precautionary measures. It may well be that participants' existing daily practices were similar to actions they needed to take for precautions against COVID-19, allowing people to accommodate precautionary behaviors without serious inconvenience. Additionally, it may well be that they viewed the measures as not presenting a significant intrusion on their lives. Certainly, it is the case that mask-wearing when sick is a common practice in Japan (and other parts of East Asia), which would mitigate against resistance to or a feeling of inconvenience related to mask-wearing within the context of the pandemic. Regardless, these results suggest that the impact of prior mindset and existing lifestyle on COVID-19 should be further explored.

It is interesting that although Iwate has few COVID-19 cases, and none at the time of focus group session, the preventative measures detailed by the participants appear to be just as thorough as the measures being taken in other places, such as Tokyo, which have had far more cases. Participants shared experiences in nursing care services, in which the staff follow strict protocols, including temperature monitoring, using personal protective equipment, and careful adherence to regulations for closing facilities. These measures are consistent with efforts to protect older people using care services in other parts of the world where COVID-19 is more widespread, like the United States (Chen et al. 2020). However, one idea that was raised 
by some participants is that the effort to follow these measures may be even greater for individuals in Iwate, as local residents may well be motivated to keep their case numbers low. In several casual conversations (via email and Zoom) with other Japanese living in the same area, a point was made that there was a certain pride in the fact that Iwate had experienced no cases and newspaper articles contemplated why the prefecture was doing so well in fighting the virus. Indeed, according to one individual an article in the main newspaper for the region claimed that the success of Iwate in avoiding the virus might be related to the fact that the prefectural governor is a graduate of Johns Hopkins University. This sense of exceptionalism related to the experience of COVID in Iwate, however, has not been entirely positive. One restaurant owner indicated that she had to make signs to place on cars with out-ofprefecture license plates indicating that the owner was actually an Iwate resident. This was in response to reports of negative comments aimed at outsiders who were perceived as potentially bringing the virus to Iwate.

One point raised as participants described their ideas about successful responses to the pandemic in Japan is the widespread use of home help services, in which careful protective measures are taken. In home help services, the goal is to support the older people so that they can continue living independently in their own homes, so nurses visit older people at their homes and help with daily tasks. We would suggest that during the pandemic, widespread use of home help services may be particularly important in relation to overall elder health as it relates to the pandemic, because older people, at least in Iwate, tend to stay home. Hence, there is assurance of regular health-checks when home helpers visit their clients. Also, because Japan's elder care system has included home help services for many years, this existing service alleviates some of the logistical obstacles of remaining at home during COVID19, such as going out to buy groceries or pick up medicine. In other words, the widespread presence of home help services has meant that a solid infrastructure was already in place to address the needs of home-bound elders prior to the pandemic.

However, home help services providing the support for older people to stay home to avoid COVID-19 does not mean the consequences of doing so are dismissed. The sacrifices to health, both mental and physical, that older individuals make in order to practice social distancing have been noted in academic literature and were reflected in the comments of people who participated in our study (Sepulveda-Loyola et al. 2020). Participants made note of physical impacts that are consistent with previous studies, like lack of exercise (Goethals et al. 2020), which has resulted in physical difficulties such as increased joint pain. Regarding mental health impacts, studies on this subject reference depression, stress, and anxiety (Banerjee 2020; Meng et al. 2020), while the impacts mentioned by participants in this study were mainly obstacles to maintaining social and family relationships, which could lead to mental health issues that were not uncovered in this research due to limited engagement timeframe.

Lastly, despite acknowledging these consequences, participants seemed to understand the necessity of the stringent measures to take against COVID-19 when it comes to the elderly. From this, it can be understood that the participants viewed protecting older people from COVID-19 as a top priority. A discrepancy to note is that despite most of the participants themselves being in the age range 
deemed at high risk for COVID-19 (World Health Organization 2020), many referenced elder individuals they knew, and only one participant mentioned the impact of social distancing on themselves. It could be that these participants do not view themselves as elderly or make a distinction based on perceived health of themselves versus others. In Japan, people in their $60 \mathrm{~s}$ are usually still relatively active, so they generally have high self-perceived health. Indeed, the participants were able to go out to this class (all wore masks), and the ability to participate in activities like this has been observed to relate to higher subjective ratings of personal health (Sun et al. 2020). Perceptions related to pre-existing health conditions and vulnerability to COVID-19 based on elder age groups could well be different among countries, which is another topic warranting further study.

Some research attention has focused on examining the impacts of cultural differences related to the experience of COVID-19 (Messner 2020; Huynh 2020). In our study, participants expressed that certain traits may contribute to how individuals handle and respond to COVID-19. One common theme was the relationship with others in society, specifically the effort people make to avoid burdening others. Participants mentioned both attentiveness to this concept and actions that reflect it, like not going out at night or to parties. Here especially, the fact that our research was limited to the older age group should be emphasized, as younger adults in Japan, or people in general from other parts of Japan, may have different views and values as compared to those who participated in this study (Elliott et al. 2012).

Nevertheless, participants in this study highlighted traits that are related to Japanese norms associated with acting responsibly in society and that encourage burden avoidance. During the pandemic, the public discourse in Japan has largely presented citizens' sense of responsibility in society as being a key element in preventing the spread of COVID-19. Doing so requires following precautionary measures, often set by authorities in government and medical organizations, and people may be more attuned to the expectations of authorities, which is reflected in the participants' inclination to follow the rules. Participants also believed that other shared traits play a role in the local response to COVID-19. The use of "we" and "people" was common when describing these traits, indicating that these behaviors may be perceived as being a specific characteristic of either those living in Iwate or Japanese people more generally. A value that was particularly evident was the attention to cleanliness, which is also tied to the value of burden avoidance. It has been noted that during influenza season, Japanese people routinely practice the added caution of wearing masks when sick (as a means to prevent spread to others) or when healthy to avoid contracting illness, which could be related to practicing additional preventative measures, like avoiding crowds and washing hands (Wada et al. 2012). Masks have also been viewed as extending beyond usage during flu season to providing a general sense of protection (Burgess and Horii 2012). This existing effort to practice good hygiene as a promotion of health seems likely to support widespread practice of preventative measures against COVID-19, as the general public is already accustomed to doing so for other, less serious, illnesses. For Iwate specifically, many participants noted their natural environment when discussing possibilities for the lack of COVID-19 cases, specifically the clean air, which ties-in to cultural values 
associated with cleanliness, and as clean air is perceived an attribute of Iwate, Iwate could be viewed as a sanctuary from COVID-19.

Lastly, participants took into account past experiences, from the immediate context of Iwate to Asia more generally. The Iwate people's experience of the Great East Japan Earthquake is particularly worth noting. Occurring in 2011, the 9.0 magnitude earthquake and resulting tsunami contributed to over 15,000 lives lost, with a disproportionate amount being elderly, and over 16 trillion yen in damage (Mimura et al. 2011). It has been noted that countries recently experiencing epidemics may have been better equipped for COVID-19. For example, after the 2003 SARS outbreak, the Taiwan government established the National Health Command Center, which in the present pandemic, has been quick to mobilize efforts to mitigate COVID-19 (Wang et al. 2020a, b). However, the Great East Japan Earthquake, recent enough that individuals still remember experiencing its devastating impact firsthand, shows that other crises could also play a role in COVID-19. From the participants' view, it's not necessarily that the government's emergency response is effective and prepared - albeit that still seems likely to play a role — but more that the people themselves already have firsthand experience with a disaster. Although the experience differs by person, it instills a collective sense of crisis, which may drive individuals to be more cautious in order to prevent another disaster.

\section{Concluding Thoughts}

Our research provides insight into the ways in which older people in an area that has only been affected by COVID-19 to a limited extent, intertwine perceptions related to their environment, culture, and social norms as they interpret their situations and experiences. This provides a starting point for developing an understanding of the lived experience of older people in the context of the pandemic and also, with further research, may provide insight into the ways in which older people think about strategies to cope with and mitigate the spread of COVID-19. It is also necessary to acknowledge the variations between groups of people in relation to factors such as age, gender, race, and ethnicity. This study focuses on the perceptions of older adults living in one city in northern Japan, and younger generations in Japan-even in the same city-may have different ideas related to both the experience and response to COVID-19 (Elliott et al. 2012).

In this study, we described the perceptions, thoughts, and experiences related to COVID-19 among older individuals living in Iwate, Japan, an area that did not confirm any COVID-19 cases until late July of 2020, through thematic analysis. It was found that individuals took into account their previous experiences and cultural values and practices when considering the current situation. Individuals also referenced caution and thoroughness in current actions against COVID-19. These findings suggest that despite living with few cases of COVID-19, people in the area continue to maintain high levels of awareness about the pandemic and feel its impact on their lives. Although a subtle point, this may well be a significant factor in stemming the spread of the virus in the region. Furthermore, we reveal that existing behaviors and values are included in people's thoughts and actions of the present, making personal 
and social context important to consider in policy making or when studying differences between groups of people. Lastly, we highlight the need to specifically look into the contexts of older adults and other vulnerable populations in order to gain a deeper understanding of how the pandemic has changed their lives and find the most effective ways to support them during this time.

\section{Postscript}

In the six months since research for this paper was completed, much has happened internationally in relation to the COVID-19 pandemic as well as within Japan. The first positive case in Iwate Prefecture was confirmed shortly after completion of our study. Between 27 July 2020 (when the first case was reported in the prefecture) and 10 January 2021, there have been a total of 434 cases in Iwate (with a population of 1.2 million), with 60 hospitalizations and 25 deaths. Since a high point of just under 100 people hospitalized in mid-December, the prefecture has averaged roughly 55 people hospitalized daily in the month of January. The city of Ōshū has remained an area of the prefecture with among the fewest cases, with a total of only six positive cases since the pandemic began. For detailed information about COVID-19 in Iwate Prefecture in English, readers should consult The COVID-19 informational website for the prefecture at https://iwate.stopcovid19.jp/en/.

Funding This study received no funds to support the research.

Declarations

Conflict of Interest The authors declare that they have no conflict of interest.

\section{References}

Armitage, R., \& Nellums, L. B. (2020). COVID-19 and the consequences of isolating the elderly. The Lancet Public Health, 5(5), e256. https://doi.org/10.1016/s2468-2667(20)30061-x.

Banerjee, D. (2020). The impact of Covid-19 pandemic on elderly mental health. International Journal of Geriatric Psychiatry. https://doi.org/10.1002/gps.5320.Advanceonlinepublication.10.1002/ gps.5320.

Barber, S. J., \& Kim, H. (2021). COVID-19 Worries and behavior changes in older and younger men and women. The Journals of Gerontology: Series B, 76(2), e17-e23. https://doi.org/10.1093/geron b/gbaa068.

Burgess, A., \& Horii, M. (2012). Risk, ritual and health responsibilisation: Japan's 'safety blanket' of surgical face mask-wearing. Sociology of Health \& Illness, 34(8), 1184-1198. https://doi.org/10.111 1/j.1467-9566.2012.01466.x.

Chen, A. T., Ryskina, K. L., \& Jung, H.-Y. (2020). Long-Term Care, Residential Facilities, and COVID19: An Overview of Federal and State Policy Responses. Journal of the American Medical Directors Association, 21(9), 1186-1190. https://doi.org/10.1016/j.jamda.2020.07.001.

Di Renzo, L., Gualtieri, P., Pivari, F., Soldati, L., Attinà, A., Cinelli, G., et al. (2020). Eating habits and lifestyle changes during COVID-19 lockdown: an Italian survey. Journal of Translational Medicine, 18(1), 229. https://doi.org/10.1186/s12967-020-02399-5. 
Elliott, A., Katagiri, M., \& Sawai, A. (2012). The New Individualism and Contemporary Japan: Theoretical Avenues and the Japanese New Individualist Path. Journal for the Theory of Social Behaviour, 42(4), 425-443. https://doi.org/10.1111/j.1468-5914.2012.00496.x.

Goethals, L., Barth, N., Guyot, J., Hupin, D., Celarier, T., \& Bongue, B. (2020). Impact of Home Quarantine on Physical Activity Among Older Adults Living at Home During the COVID-19 Pandemic: Qualitative Interview Study. JMIR Aging, 3(1), e19007. https://doi.org/10.2196/19007.

Guo, T., Shen, Q., Guo, W., He, W., Li, J., Zhang, Y., Wang, Y., Zhou, Z., Deng, D., Ouyang, X., Xiang, Z., Jiang, M., Liang, M., Huang, P., Peng, Z., Xiang, X., Liu, W., Luo, H., Chen, P., Peng, H. (2020). Clinical characteristics of elderly patients with COVID-19 in Hunan Province, China: a multicenter, retrospective study. Gerontology, 66(5), 467-475. https://doi.org/10.1159/000508734.

Huynh, T. L. D. (2020). Does culture matter social distancing under the COVID-19 pandemic? Safety Science, 130, 104872. https://doi.org/10.1016/j.ssci.2020.104872.

Ikegami, N. (1991). Japanese Health Care: Low Cost Through Regulated Fees. Health Affairs, 10(3), 87-109. https://doi.org/10.1377/hlthaff.10.3.87.

Japan Health Policy NOW - Health Insurance System. (n.d.). Japan Health Policy Now. http://japanhpn. org/en/hs1/.

Khafaie, M. A., \& Rahim, F. (2020). Cross-Country Comparison of Case Fatality Rates of COVID19/SARS-COV-2. Osong Public Health and Research Perspectives, 11(2), 74-80. https://doi. org/10.24171/j.phrp.2020.11.2.03.

Liu, K., Chen, Y., Lin, R., \& Han, K. (2020). Clinical features of COVID-19 in elderly patients: A comparison with young and middle-aged patients. Journal of Infection, 80(6), e14-e18. https://doi. org/10.1016/j.jinf.2020.03.005.

Liu, Q., Luo, D., Haase, J. E., Guo, Q., Wang, X. Q., Liu, S., et al. (2020). The experiences of health-care providers during the COVID-19 crisis in China: a qualitative study. The Lancet Global Health, 8(6), e790-e798. https://doi.org/10.1016/s2214-109x(20)30204-7.

Lock, M. (1995). Encounters with Aging: Mythologies of Menopause in Japan and North America. Berkeley: University of California Press.

Mathews, G. (1996). The Stuff of Dreams, Fading: Ikigai and" The Japanese Self". Ethos, 24(4), 718-747.

Matsuyama, Y. (2014). Aging and the governance of the healthcaresystem in Japan, Bruegel Working Paper, No. 2014/14, Bruegel, Brussels.

Meng, H., Xu, Y., Dai, J., Zhang, Y., Liu, B., \& Yang, H. (2020). Analyze the psychological impact of COVID-19 among the elderly population in China and make corresponding suggestions. Psychiatry Research, 289, 112983. Advance online publication. https://doi.org/10.1016/j.psychres.2020.11298 3.

Messner, W. (2020). The Institutional and Cultural Context of Cross-National Variation in COVID-19 Outbreaks. Medrxiv.

Mimura, N., Yasuhara, K., Kawagoe, S., et al. (2011). Damage from the Great East Japan Earthquake and Tsunami - A quick report. Mitigation and Adaptation Strategies for Global Change, 16, 803-818. https://doi.org/10.1007/s11027-011-9297-7.

Munawar, K., \& Choudhry, F. R. (2021). Exploring stress coping strategies of frontline emergency health workers dealing Covid-19 in Pakistan: A qualitative inquiry. American Journal of Infection Control. (in press)

National Institute of Population and Social Security Research. (2018). Regional Population Projections for Japan: 2015-2045. http://www.ipss.go.jp/pp-shicyoson/e/shicyoson18/t-page.asp.

Niu, S., Tian, S., Lou, J., Kang, X., Zhang, L., Lian, H., \& Zhang, J. (2020). Clinical characteristics of older patients infected with COVID-19: A descriptive study. Archives of Gerontology and Geriatrics, 89, 104058. https://doi.org/10.1016/j.archger.2020.104058.

Ohnuki-Tierney, E. (1984). Illness and culture in contemporary Japan: An anthropological view. New York: Cambridge University Press.

Patel, S., \& Clark-Ginsberg, A. (2020). Incorporating Issues of Elderly Loneliness into the Coronavirus Disease-2019 Public Health Response. Disaster Medicine and Public Health Preparedness, 1-2. https://doi.org/10.1017/dmp.2020.145

Sasaki, N., \& Nishizuka S.S. (2020) Affected Medical Services in Iwate Prefecture in the Absence of a COVID-19 Outbreak. medRxiv 2020.06.19.20135269. https://doi.org/10.1101/2020.06.19.20135 269.

Sepulveda-Loyola, W., Rodriguez-Sanchex, I., Perez-Rodriguez, P., Ganz, F.; Torralba, R., Oliviera, D.V., Rodriguez-Manas, L. (2020). Impact of social isolation due to Covid-19 on health in older people: 
Mental and physical effects and recommendations. The Journal of Nutrition Health and Aging, 110. https://doi.org/10.1007/s12603-020-1469-2.

Sun, N., Wei, L., Shi, S., Jiao, D., Song, R., Ma, L., et al. (2020). A qualitative study on the psychological experience of caregivers of COVID-19 patients. American Journal of Infection Control, 48(6), 592-598. https://doi.org/10.1016/j.ajic.2020.03.018.

Sun, W., Watanabe, M., Tanimoto, Y., Shibutani, T., Kono, R., Saito, M., et al. (2007). Factors associated with good self-rated health of non-disabled elderly living alone in Japan: a cross-sectional study. BMC Public Health, 7, 297. https://doi.org/10.1186/1471-2458-7-297.

Traphagan, J. W. (2000). Taming Oblivion: Aging Bodies and the Fear of Senility in Japan. Albany: State University of New York Press.

Traphagan, J. W. (2004a). The Practice of Concern: Ritual, Well-Being, and Aging in Rural Japan. Durham: Carolina Academic Press.

Traphagan, J. W. (2004b). Culture and Long Term Care: The Bath as Social Service in Japan. Care Management Journals, 5(1), 53-60.

Traphagan, J. W., \& Nagasawa, T. (2008). Group Homes for Elders with Dementia in Japan. Care Management Journals, 9(2), 89-96.

Traphagan, J. W. (2020). Cosmopolitan Rurality, Depopulation, and Entrepreneurial Ecosystems in $21^{\text {st }}$ Century Japan Century Japan. Amherst, NY: Cambria Press.

Traphagan, J. W., \& Knight, J. (2003). Demographic Change and the Family in Japan's Aging Society. Albany, NY: SUNY Press.

Tsukada, N., \& Saito, Y. (2006). Factors that affect older Japanese people's reluctance to use home help care and adult day care services. Journal of Cross-Cultural Gerontology, 21(3-4), 121-137.

Wada, K., Oka-Ezoe, K., \& Smith, D. R. (2012). Wearing face masks in public during the influenza season may reflect other positive hygiene practices in Japan. BMC Public Health, 12, 1065. https://doi. org/10.1186/1471-2458-12-1065.

Wand, A., Zhong, B., Chiu, H., Draper, B., \& De Leo, D. (2020). COVID-19: the implications for suicide in older adults. International Psychogeriatrics, 1-6. https://doi.org/10.1017/S1041610220000770.

Wang, C. J., Ng, C. Y., \& Brook, R. H. (2020). Response to COVID-19 in Taiwan. JAMA, 323(14), 1341-1342. https://doi.org/10.1001/jama.2020.3151.

Wang, L., He, W., Yu, X., Hu, D., Bao, M., Liu, H., et al. (2020). Coronavirus disease 2019 in elderly patients: Characteristics and prognostic factors based on 4-week follow-up. Journal of Infection, 80(6), 639-645. https://doi.org/10.1016/j.jinf.2020.03.019.

World Health Organization. (2021). WHO Coronavirus Disease (COVID-19) Dashboard. https://covid 19.who.int/.

World Health Organization. (2020). COVID-19 High risk groups. https://www.who.int/westernpacific/ emergencies/covid-19/information/high-risk-groups.

Zhu, N., Zhang, D., Wang, W., Li, X., Yang, B., Song, J., et al. (2020). A Novel Coronavirus from Patients with Pneumonia in China, 2019. New England Journal of Medicine, 382(8), 727-733. https ://doi.org/10.1056/nejmoa2001017.

Zuo, P., Tong, S., Yan, Q., Cheng, L., Li, Y., Song, K., Chen, Y., Dai, Y., Gao, H., \& Zhang, C. (2020). Decreased prealbumin level is associated with increased risk of mortality in hospitalized elderly patients with COVID-19. Nutrition, 78, 110930. https://doi.org/10.1016/j.nut.2020.110930. 